\title{
Absorption spectrum of monomeric pseudoisocyanine: A new perspective and its implications for formation and spectral response of $\mathrm{J}$-aggregates in solution and in thin films
}

\author{
Demet Gülen ${ }^{\mathrm{a}, *, 1}$, Serdar Özçelik ${ }^{\mathrm{b}}$ \\ ${ }^{a}$ Physics Department, Middle East Technical University (METU), 06531 Ankara, Turkey \\ ${ }^{\mathrm{b}}$ Chemistry Department, İmir Institute of Technology, Urla 35430, İmir, Turkey
}

Available online 6 November 2007

\begin{abstract}
We argued against the current spectral assignment for absorption spectrum of monomeric PIC which is widely accepted since the pioneering works of Scheibe and Jelley [G. Scheibe, Angew. Chem. 49 (1936) 563; E.E. Jelly, Nature 138 (1936) 1009]. A new spectrum is presented along with its conceptual basis. The hypothesized spectrum attributes the previous $0-0(\approx 525 \mathrm{~nm})$ and $0-1(\approx 490 \mathrm{~nm})$ assignments, respectively, to intermediates acting as the precursor of J-aggregates and to the $0-0$ transition of monomeric PIC and brings the spectrum in accord with the seemingly universal spectral fingerprint of cyanines. The hypothesis is used to analyze and interpret the temperature dependence of the UV-vis absorption of PIC aggregates in saline aqueous solution by incorporating the J-band simulations within frenkel exciton formalism. Its implications for aggregate formation kinetics are given on the basis of current spectroscopic evidence. The hypothesis readily answers several long-standing questions: Why compared to many other cyanines at least an order of magnitude higher dye concentration is needed to form J-aggregates of PIC? Why are there no precursors, since aggregation is expected to be a consecutive process? A large number of observations on steady-state and time-resolved spectral properties, and aggregation kinetics in solution/thin films are likely to find reasonable explanations within this hypothesis.
\end{abstract}

(C) 2007 Elsevier B.V. All rights reserved.

Keywords: UV-vis absorption; Pseudoisocyanine; J-aggregates; Frenkel exciton

\section{Introduction}

A prominent, narrow and strongly red-shifted band has been the signature for J-aggregate formation by numerous cyanines since their discovery by Jelly [1] and Scheibe [2]. Despite extensive investigation, the spectral changes following self-aggregation and the kinetics of aggregate formation in solution/thin films are still debated [3-8].

The Frenkel exciton picture [9] is well agreed as the basis for understanding their optical response $[3-8,10]$. The prerequisite to reveal the aggregate optical response within the Frenkel picture is a well-defined monomeric absorption spectrum. The spectrum in water at highly dilute dye concentration and moderate temperatures is commonly

\footnotetext{
*Corresponding author. Fax: + 903122105099

E-mail address: dgul@metu.edu.tr (D. Gülen).

${ }^{1}$ On leave at METU/Northern Cyprus Campus.
}

assumed to represent the absorption of monomeric PIC [6-8]. Under these conditions the absorption is characterized by two peaks $\approx 525$ and $\approx 490 \mathrm{~nm}$, and a shoulder $\approx 460 \mathrm{~nm}$. The $525 \mathrm{~nm}$ peak is associated with the $0-0$ transition while the 490 and $460 \mathrm{~nm}$ transitions are attributed to vibrational bands. We, however, note that this spectral shape does not share the seemingly universal fingerprint of cyanines which consists of a $0-0$ transition and a "single" $(0-1)$ vibrational shoulder [3-5]. Consequently, we have questioned this assignment which is widely accepted as a fact, while it is only a proposition which relies on the assumption that the PIC is monomeric under the conditions stated above.

\section{Theoretical methods}

The Frenkel exciton formalism [9] augmented by diagonal energetic disorder [10] and exciton-phonon 
coupling [11] is used to simulate the J-band. We give an indepth account of the formalism and its numerical execution in Ref. [5]. To provide a structural model has not been our purpose. We merely used the simple linear chain for the sake of having a model to simulate the J-band shape/width. The simulations are ensemble averages over $10^{3}$ chains. The simulation parameters are: $N$, the number molecules/ chain; $E_{0-0}$, the $0-0$ transition energy; $\sigma$, the full width at half maximum (FWHM) of the Gaussian distribution representing diagonal disorder; $\hbar S$, the exciton-phonon coupling parameter; $V_{\mathrm{nn}}$, the nearest-neighbor interaction and $T$, temperature. $V_{\mathrm{nn}}, \sigma$ and $\hbar S$ are the fit parameters.

\section{Results and discussion}

Fig. 1 displays a set of absorption spectra in saline aqueous solution at "high PIC concentration" at different $T$ between 15 and $55^{\circ} \mathrm{C}$, where $T^{\circ} \mathrm{C}$ indicates temperature in centigrades. The low concentration spectrum of the inset is typical of the spectra commonly assigned to monomeric PIC. Several features to note are: the high $T$ spectrum highly resembles the alleged monomeric spectrum; a typical J-band $(\approx 574 \mathrm{~nm})$ is exhibited upon cooling whose position is independent of $T$; its intensity increases at decreasing $T$; the blue band hosting the $490 \mathrm{~nm}$ peak and the $460 \mathrm{~nm}$ shoulder experiences an overall red-shift upon decreasing $T$; the amount of red-shift from 55 to $15^{\circ} \mathrm{C}$ is $\approx 5 \mathrm{~nm}$; and the $490 \mathrm{~nm}$ peak becomes sharper at lower $T$.

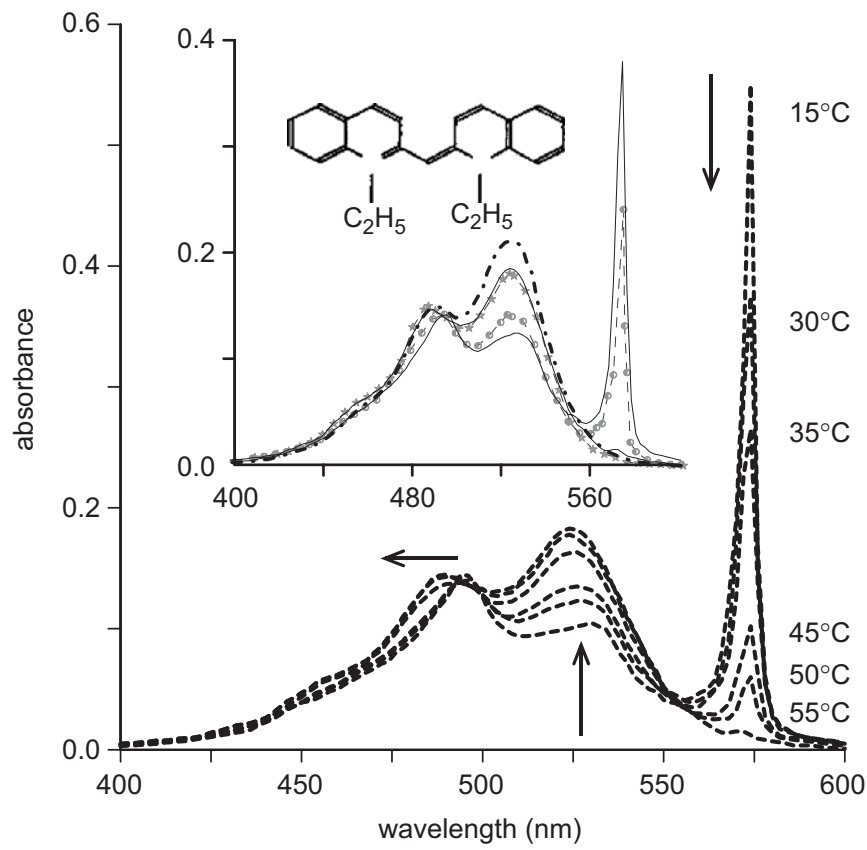

Fig. 1. Absorption spectra of PIC in saline aqueous solution $(150 \mu \mathrm{M}$ in $5 \mathrm{M} \mathrm{NaCl} / \mathrm{H}_{2} \mathrm{O}$ ) at different temperatures (reproduced from Refs. [7,8]). The inset (arbitrarily scaled) shows the absorption spectrum of PIC $\left(23.5 \mu \mathrm{M}\right.$ ) in water at $21^{\circ} \mathrm{C}$ (dash-dot, and the $30^{\circ} \mathrm{C}$ (lower) and $55^{\circ} \mathrm{C}$ (upper) data of the main figure (solid lines); symbols (the 80 and $20^{\circ} \mathrm{C}$ data of Ref. [8], $300 \mathrm{~mol} / \mathrm{L} \mathrm{PIC,} \mathrm{in} 0.2 \mathrm{~mol} / \mathrm{L} \mathrm{H}_{2} \mathrm{O} / \mathrm{NaCl}$ solution). The $400-450 \mathrm{~nm}$ tails of the data of Ref. [8] are used to extrapolate the data of Ref. [7].
We start our discussion by suggesting that the $525 \mathrm{~nm}$ band is not monomeric, but it may be originating from some intermediates which serve as the seed of J-aggregates forming the $574 \mathrm{~nm}$ band. A symmetric Gaussian (peak $=524 \mathrm{~nm}, \mathrm{FWHM}=550 \mathrm{~cm}^{-1}$ ) fits the $525 \mathrm{~nm}$ band well, especially at high $T$. Fig. 2a shows the residual upon subtracting such a Gaussian of adjusted intensity from the $55^{\circ} \mathrm{C}$ spectrum. The comparison given in Fig. $2 \mathrm{~b}$ clearly illustrates that this residual $(\leqslant 525 \mathrm{~nm})$ assumes the spectral fingerprint common to a large number of cyanines, including those with very complex structures [3]. Considering this similarity, we assign the residual to the absorption spectrum of monomeric PIC and attribute the $490 \mathrm{~nm}$ peak to the $0-0$ and the $460 \mathrm{~nm}$ shoulder to the $0-1$ vibrational transitions.

Next we interpret the $T$ dependence of the aggregate spectra of Fig. 1 within our hypothesis. Fig. 3 contains the resolution of the experimental data. A weighted super-
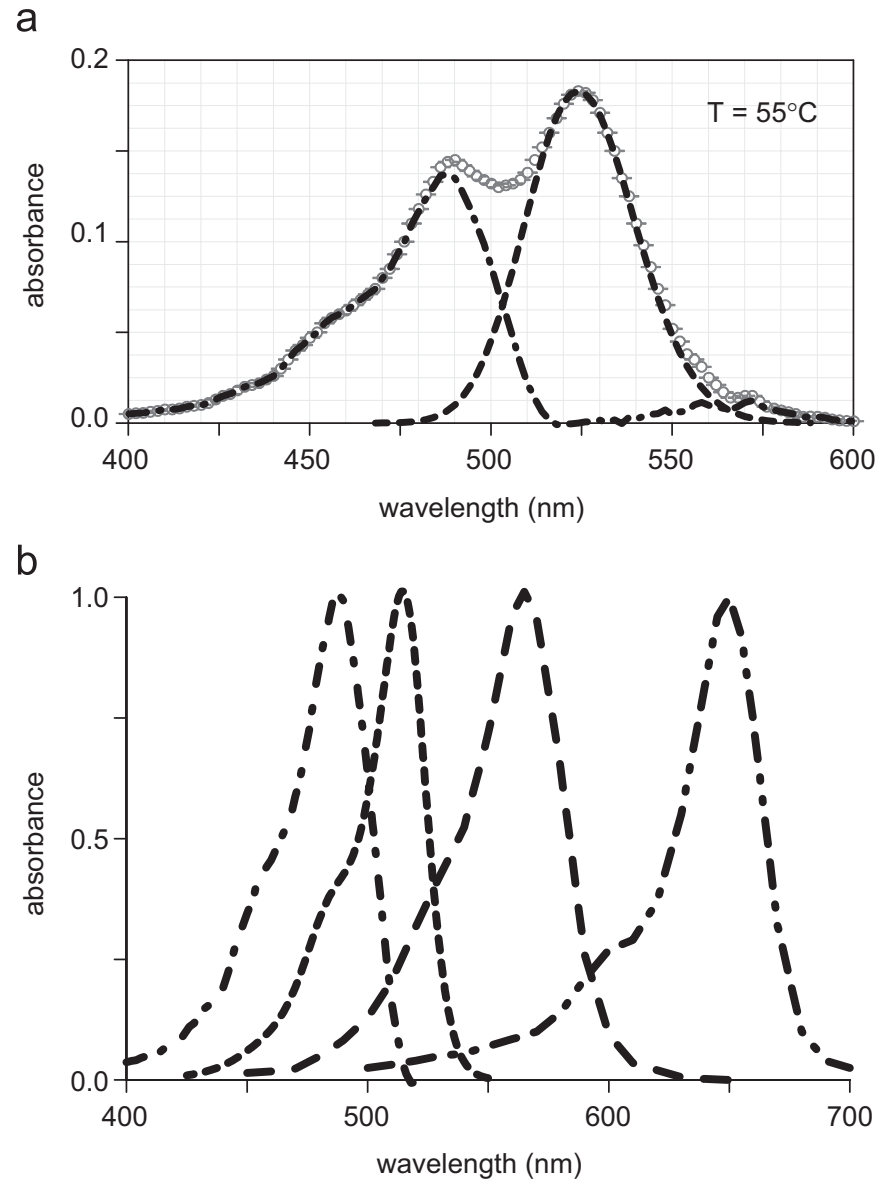

Fig. 2. (a) The experimental data at $55^{\circ} \mathrm{C}$ (symbols); the Gaussian band, peak $=524 \mathrm{~nm}, \mathrm{FWHM}=550 \mathrm{~cm}^{-1}$ (dashes); the residual (dash-dot). (b) The hypothesized spectrum versus the monomeric absorption of several cyanines, normalized at their peak of $0-0$ transition (from left to right, PIC, TTBC, 'T307', 'DyeIII'). TTBC (1,1',3,3'-tetraethyl-5,5',6,6'-tetrachlorobenzimidazolocarbocyanine [5]); 'T307' (bis-[3-ethyl-6 diethylaminobenzothiazole(2)]-trimethine cyanine iodide-6,6-benzoyl-amino substituted with methylorthocarbonate [3]); 'DyeIII' (3-ethyl-2-[5-(3ethyl-3H-benzothiazol-2-ylidene)-3-methylpenta-1,3-di-enyl] benzothiazolium-p-toluenesulfonate, [4]). 

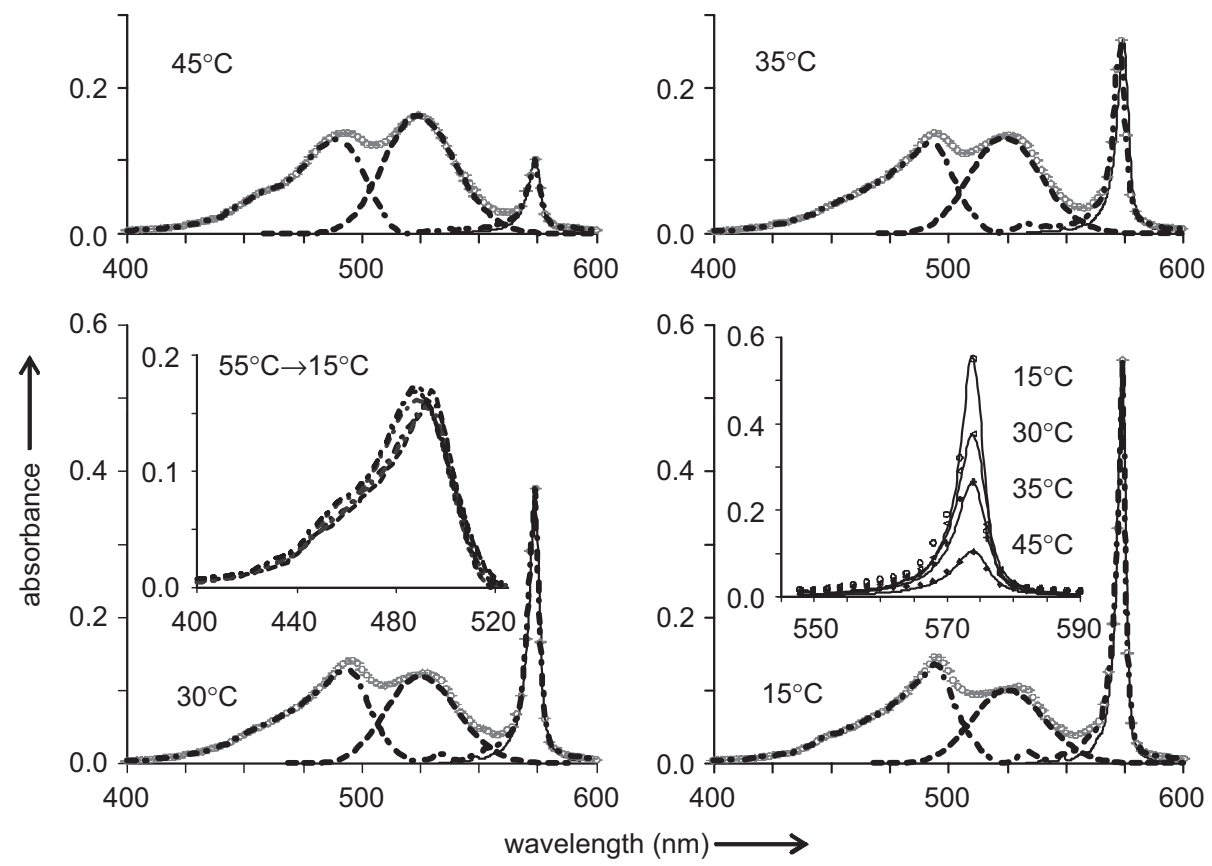

Fig. 3. Resolution of the experimental data. Dashed (the Gaussian, peak $=524 \mathrm{~nm}, \mathrm{FWHM}=550 \mathrm{~cm}^{-1}$ at all $T$ ); dash-dot (the residual: the experimental data (symbols) minus the Gaussian); solid (the J-band simulations). Inset, the residuals between 15 and $55^{\circ} \mathrm{C}$ (from right to left). Inset, the residuals (symbols) and the J-band simulations (solid). $N=50, V_{\mathrm{nn}}=1266 \mathrm{~cm}^{-1}, E_{0-0}=20,492 \mathrm{~cm}^{-1} 488 \mathrm{~nm}^{-1}, \sigma=190 \mathrm{~cm}^{-1}, \hbar S$ in $\mathrm{cm}^{-1} /$ at $T^{\circ} \mathrm{C} ; 500 / 45^{\circ} \mathrm{C}, 525 /$ $35^{\circ} \mathrm{C}, 550 / 30^{\circ} \mathrm{C}, 750 / 15^{\circ} \mathrm{C}$.

position of the monomeric absorption, the $524 \mathrm{~nm}$ Gaussian and the $574 \mathrm{~nm} \mathrm{~J}$-band, reproduce the experimental data satisfactorily. The J-band formation is accounted to a large extent by the loss in $525 \mathrm{~nm}$ absorbance. The monomeric contribution remains unchanged at high $T$ $\left(\geqslant 40{ }^{\circ} \mathrm{C}\right)$. The low $T$ residuals are likely to enclose an additional band $\approx 495 \mathrm{~nm}[7,8]$. In addition, the hypothesized spectrum is not expected to be extremely temperature dependent [6]. Therefore, at low $T$ further conversion of monomers to J-aggregates is implied. The progressive redshift $\left(\approx 5 \mathrm{~nm}\right.$ from 55 to $15^{\circ} \mathrm{C}$ ) and the enhancement of $490 \mathrm{~nm}$ intensity at low $T$ can be attributed to enhance contribution of the band $\approx 495 \mathrm{~nm}$. The existence of a weak residual band $\approx 535 \mathrm{~nm}$ also agrees with the earlier work [6-8].

\section{Concluding remarks}

Our hypothesis brought the PIC spectrum in accord with the seemingly universal spectral fingerprint of cyanine molecules which consists of a $0-0$ transition and a $0-1$ vibrational shoulder. A $0-0$ transition around $490 \mathrm{~nm}$ rules out the existence of highly debated $\mathrm{H}$-aggregates under the $525 \mathrm{~nm}$ band [7,8]. The J-aggregate nature of the $525 \mathrm{~nm}$ intermediates that already appear at low PIC concentrations readily answers the long-standing questions we have posed in the abstract. Our alternative explanation of the UV-vis spectra suggests a simple scheme for the aggregation kinetics; the monomers and the intermediates coexist in equilibrium and self-aggregation of the intermediates leads to $\mathrm{J}$-aggregate formation upon decrease of temperature.

The simple linear chain model and phenomenological dynamical picture of Ref. [11] have been merely used for obtaining reasonable simulations of $\mathrm{J}$-band. The assessment of structure-spectroscopy-dynamics relationship remains a challenge through which the mechanism of excited state dynamics could also be questioned further using a recent formalism [12].

The experimental techniques that are powerful for analyzing complicated systems with two or more spectral components such as two-dimensional excitation-emission/ fluorescence correlation spectroscopies [13] could be useful for further testing of our hypothesis.

\section{Acknowledgments}

We thank to Prof. T. Kobayashi and Prof. J. Knoester for their valuable suggestions.

\section{References}

[1] E.E. Jelly, Nature 138 (1936) 1009.

[2] G. Scheibe, Angew. Chem. 49 (1936) 563.

[3] V.B. Kovalska, K.D. Volkova, M.Y. Losytskyy, O.I. Tolmachev, A.O. Balanda, S.M. Yarmoluk, Spectrochim. Acta A 65 (2006) 271 (and the references therein).

[4] I.O. Shklyarevskiy, P.C.M. Christianen, E. Aret, H. Meekes, E. Vlieg, G. Deroover, P. Callant, L. van Meervelt, J.C. Maan, J. Phys. Chem. B 108 (2004) 16386

[5] B. Birkan, D. Gülen, S. Özçelik, J. Phys. Chem. B 110 (2006) 10805.

[6] I. Renge, U.P. Wild, J. Phys. Chem. A 101 (1997) 7977. 
[7] R.F. Khairutdinov, N.J. Serpone, Phys. Chem. B 101 (1997) 2602.

[8] I. Struganova, J. Phys. Chem. A 104 (2000) 9670 (and the references therein).

[9] R.S. Knox, Theory of Excitons, Academic Press, New York, 1963.

[10] H. Fidder, J. Knoester, D.A. Wiersma, J. Chem. Phys. 95 (1991) 7880.
[11] J.A. Leegwater, J.A. Durrant, D.A. Klug, J. Phys. Chem. B 101 (1997) 7205.

[12] D.J. Heijs, V.A. Malyshev, J. Knoester, J. Chem. Phys. 123 (2005) 144507.

[13] B. Valeur, Molecular Fluorescence: Principles and applications, Wiley-VCH, Weinheim, 2001. 